\title{
Differential expression profiling of matrix metalloproteinases and tissue inhibitors of metalloproteinases in females with or without pelvic organ prolapse
}

\author{
XIAOHONG WANG ${ }^{1,2}$, YIQI LI ${ }^{1}$, JIE CHEN $^{2}$, XIAOLI GUO ${ }^{2}$, HAN GUAN $^{2}$ and CANDONG LI ${ }^{1}$ \\ ${ }^{1}$ Fujian University of Traditional Chinese Medicine, Fuzhou, Fujian 350122; \\ ${ }^{2}$ People's Hospital Affiliated to Fujian University of Traditional Chinese Medicine, Fuzhou, Fujian 350004, P.R. China
}

Received December 13, 2013; Accepted June 26, 2014

DOI: $10.3892 / \mathrm{mmr} .2014 .2467$

\begin{abstract}
Pelvic organ prolapse (POP) is a common disorder that can disturb the health and quality of life of females. However, the basic pathophysiology and underlying mechanism of POP are not fully understood. Matrix metalloproteinases (MMPs) and tissue inhibitors of metalloproteinases (TIMPs) have been reported to be associated with the onset and development of POP. In the present study, to characterize the differential expression profile of MMPs and TIMPs in female patients with and without POP, a total of 72 POP patients were sampled as a patient group and 72 non-POP patients that underwent hysterectomy due to benign tumors were sampled as a control group. Immunohistochemistry and polymerase chain reaction analysis were used to detect the expression levels of MMP-1, -2, -3 and -9 as well as TIMP-1 protein and mRNA in the anterior vaginal wall tissues. The expression levels of MMP-1, $-2,-3$ and -9 in the patient group were found to be significantly higher than those in the control group. By contrast, TIMP-1 expression levels in the patient group were significantly lower than those in the control group. Correlational analysis revealed a significantly positive correlation among the expression levels of MMP-2, -3 and -9 . TIMP-1 expression levels were significantly negatively correlated with the expression levels of MMP-3 and -9. In addition, the expression levels of MMP-1 exhibited a positive correlation with those of MMP-2, -3 and -9 , but a negative correlation with those of TIMP-1. The results demonstrated that the increased expression levels of MMPs and the reduced expression levels of TIMPs were directly associated with the presence of uterine prolapse, indicating that the differential expression levels of MMPs and TIMPs were correlated with the occurrence and development of POP. This data may assist in elucidating the
\end{abstract}

Correspondence to: Professor Candong Li, Fujian University of Traditional Chinese Medicine, 1 Huatuo Raod, Shangjie, Minhou, Fuzhou, Fujian 350122, P.R. China

E-mail: candongli@126.com

Key words: pelvic organ prolapse, matrix metalloproteinases, tissue inhibitors of metalloproteinases, differential expression molecular mechanism of MMP and TIMP involvement in POP, and also provide an underlying theoretical basis for the prevention and treatment of POP.

\section{Introduction}

Pelvic organ prolapse (POP) is the displacement of pelvic organs caused by various types of damage to the pelvic floor fascia and ligaments, which have failed to fully recover, or by weakened supporting structures, due to hypotonia. The predominant clinical manifestations are anterior and/or posterior vaginal wall prolapse as well as uterine prolapse (1). POP, a pelvic floor dysfunction (PFD) disorder, is a global health problem that affects $50 \%$ of parous females. It contributes to reduced quality of life and is a major reason for gynecological surgery in aging females (2). The pelvic organs are supported by pelvic floor muscles, the bony pelvis, ligaments and fascial supports. Abnormalities in the connective tissues of the pelvic support system have been suggested to contribute to the genesis of POP $(3,4)$. The exact pathophysiology and natural history of POP, however, are not fully understood.

The fascia and ligaments in the bladder and urethra are mainly composed of connective tissue, and the main structural protein is type I collagen-a heterotrimer, comprising two $\alpha-1$ and single $\alpha-2$ chains encoded by the genes COL1A1 and COL1A2, respectively (5). Collagen is also a structural protein in the extracellular matrix (ECM) and is responsible for the integrity of the pelvic floor structure (6). Recent studies have demonstrated that the content, components and ultrastructure of collagen are modified in the pelvic floor structure of POP patients (7). Abnormalities in the ultrastructure and biochemical properties of collagenous fibers have also been indicated in the occurrence of PFD. A reduction in the collagen content has been shown to be due to an increase in collagen decomposition but not to a reduction in collagen synthesis. Therefore, the accelerating degradation of collagen accounts for the reduction of total collagen content in PFD patients (8).

Matrix metalloproteinases (MMPs) and tissue inhibitors of metalloproteinase (TIMPs) are two important enzymes capable of effectively regulating collagen metabolism and other ECM degradation. MMPs are secreted as inactive pre-enzymes and are transformed into active forms following cleavage of 
a propeptide domain in the molecule (9). Among $>20$ human MMPs, MMP-1 - 3 and -9 are the key enzymes resulting in weak ductility and lack of integrity in the collagen tissues (10). TIMPs combine with the relevant MMP pre-enzymes and enzymes in active form to inhibit the production and activation of MMPs, as well as the degradation of collagen. Furthermore, an imbalance between MMPs and TIMPs in ECM has been reported to be closely associated with collagen metabolism (11).

The expression and involvement of several MMPs and TIMPs in POP patients have been determined in several studies (12-14). However, the studies have produced conflicting results regarding the contribution of MMPs and TIMPs to the clinicopathological findings and prognosis of POP patients. In the present study, immunohistochemistry and quantitative polymerase chain reaction (qPCR) were employed to detect the expression levels of MMP-1-3 and -9 as well as TIMP protein and mRNA in vaginal wall tissues from POP patients and control subjects, and the correlations among these expression levels were examined. This may aid in the elucidation of the molecular mechanisms of the involvement of MMPs and TIMP in POP, and also provide an underlying theoretical basis for the etiology, and the prevention and treatment of POP.

\section{Materials and methods}

Sample collection. The present study was reviewed and approved by the Human Ethics Committees Review Board at Fujian University of Traditional Chinese Medicine (Fuzhou, China) with written informed consent obtained from each respondent.

The subjects were 72 POP patients who received surgical treatment at the People's Affiliated Hospital of Fujian University of Traditional Chinese Medicine between September 2011 and September 2012. All prolapse cases were diagnosed and recorded according to the pelvic organ prolapse quantification score issued by the International Temperance Association in 1996. A total of 72 non-POP cases that underwent hysterectomy during the same period due to benign tumors served as a control group. All respondents were from the Fuzhou district, but of different socioeconomic backgrounds. All respondents met the following criteria: The respondents agreed to be involved in this study; the respondents were able to understand and complete the assessment scales correctly, and fill out questionnaires; and the respondents were neither taking hormonal drugs nor suffering from a functional ovarian tumor within the last three months. The patients had not undergone prior vaginal surgery. Patients with cancer, endometriosis, gynecological inflammation, connective tissue disease or other diseases associated with MMPs, in addition to those who had previously given birth by caesarian, were excluded from the present study.

Immunohistochemistry. The anterior vaginal wall tissues from the POP patients and the control group were cut into $5-\mu \mathrm{m}$ sections, placed on glass slides and fixed with $4 \%$ paraformaldehyde. The slides were then stained using an avidin-biotin complex (Thermo Fisher Scientific, Waltham, MA, USA) method. The sections were incubated with primary antibodies as follows: MMP-1, 1:50, overnight at room temperature;
Table I. Primer sequences for polymerase chain reaction.

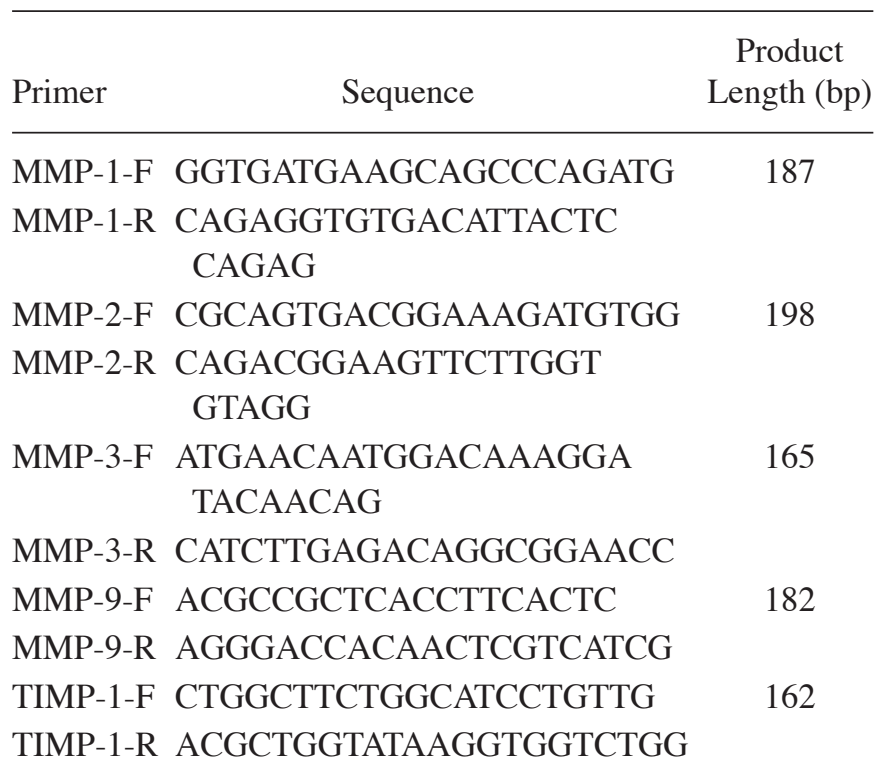

bp, base pairs; MMP, matrix metalloproteinase; TIMP, tissue inhibitor of metalloproteinase; F, forward; R, reverse.

Table II. Clinical data of the POP group and control groups.

\begin{tabular}{|c|c|c|c|}
\hline & $\begin{array}{l}\text { POP group } \\
\quad(n=72)\end{array}$ & $\begin{array}{l}\text { Control group } \\
\qquad(\mathrm{n}=72)\end{array}$ & $\begin{array}{l}\text { Mann-Whitney } \\
\text { test P-value }\end{array}$ \\
\hline Age, years & $59.01 \pm 7.657$ & $59.53 \pm 8.962$ & 0.540 \\
\hline Deliveries, n & $2.24 \pm 0.617$ & $2.29 \pm 0.615$ & 0.651 \\
\hline $\begin{array}{l}\text { Menopausal } \\
\text { status, n (\%) }\end{array}$ & $12(16.67 \%)$ & $11(15.28 \%)$ & 0.820 \\
\hline
\end{tabular}

Age and number of deliveries data are presented as the mean \pm standard deviation. POP, pelvic organ prolapse.

MMP-2, 1:50, $60 \mathrm{~min}$ at room temperature; MMP-3, 1:50, $60 \mathrm{~min}$ at room temperature; MMP-9, 1:100, $60 \mathrm{~min}$ at room temperature; and TIMP-1, 1:100, $60 \mathrm{~min}$ at room temperature (all antibodies were purchased from Thermo Fisher Scientific). Subsequent to washing with phosphate-buffered saline, biotin-labeled link secondary antibodies (1:1,000; Thermo Fisher Scientific) and streptavidin-biotin peroxidase were applied using a labeled streptavidin-biotin (LSAB) kit (Dako, Carpinteria, CA, USA). Bound peroxidase was detected by diaminobenzidine. A brown reaction product indicated a positive reaction for antibody staining.

RNA isolation and quality inspection. The anterior vaginal wall tissues from POP patients and the control group were collected during surgery. Subsequent to removal from the body, the tissue samples were immediately dissolved with RNAlater Stabilization Reagent (Qiagen, Valencia, CA, USA) and then placed in a $-80^{\circ} \mathrm{C}$ refrigerator. Total RNA from each sample was individually isolated using TRIzol (Invitrogen Life Technologies, Carlsbad, CA, USA) and a miRNeasy mini kit 


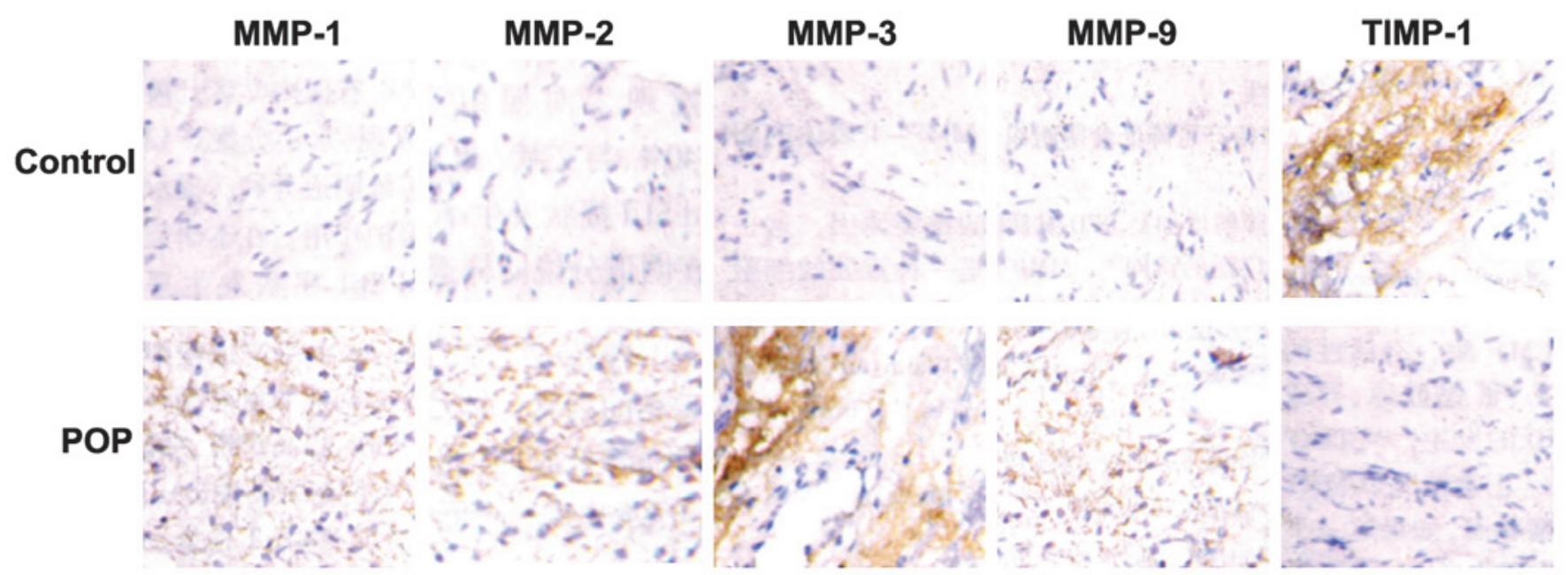

Figure 1. Immunohistochemical analysis of MMP and TIMP-1 expression in the anterior vaginal wall tissues from POP patients and the control group. Representative images show the immunohistochemistry results for immunoreactive MMP-1, MMP-2, MMP-3, MMP-9 and TIMP-1 in the anterior vaginal wall tissues. Brown staining indicated a positive reaction with the respective antibody. Original magnification, x400. MMP, matrix metalloproteinase; POP, pelvic organ prolapse; TIMP-1, tissue inhibitor of metalloproteinase 1.

(Qiagen) according to the manufacturer's instructions. RNA quality and quantity were measured with a NanoDrop spectrophotometer (ND-1000; NanoDrop Technologies, Wilmington, DE, USA), and RNA integrity was determined by gel electrophoresis imaged by Chemi Doc XRS + gel imaging system (Bio-Rad, Hercules, CA, USA).

qPCR analysis. The expression levels of MMP and TIMP mRNA were analyzed using a SYBR-based qPCR method. Briefly, 100 ng total RNA was reverse-transcribed to cDNA with gene-specific stem-loop RT primers in a Veriti Thermal Cycler Detector (Applied Biosystems, Foster City, CA, USA). qPCR was performed using Thunderbird SYBR qPCR mix (Toyobo Corporation, Osaka, Japan), according to the manufacturer's instructions, in a Mastercycler ${ }^{\circledR}$ ep Realplex PCR instrument (Eppendorf, Hamburg, Germany). The MMP and TIMP mRNA levels were normalized to those of U6, which served as an internal control. The relative abundance of each mRNA was calculated using the comparative $\mathrm{Ct}\left(2^{-\Delta \Delta C t}\right)$ method and the results were assessed by t-test. The primers are listed in Table I.

Statistical analysis. Comparisons of two-group parameters were performed using Student's t-test. Comparisons of multiple group data were performed using one-way analysis of variance followed by Turkey's post-hoc test. $\mathrm{P}<0.05$ was considered to indicate a statistically significant difference. Statistical analysis was performed using the SPSS statistical package, version 19.0 (IBM SPSS, Armonk, NY, USA).

\section{Results}

Clinical characteristics of patients. The vaginal wall tissues from the 72 female POP patients and 72 female control subjects were collected and analyzed. The median ages of the POP patients and the control group were both 59 years. The average number of deliveries of POP patients was $2.24 \pm 0.617$, whereas that of the control group was $2.29 \pm 0.615$. In the POP

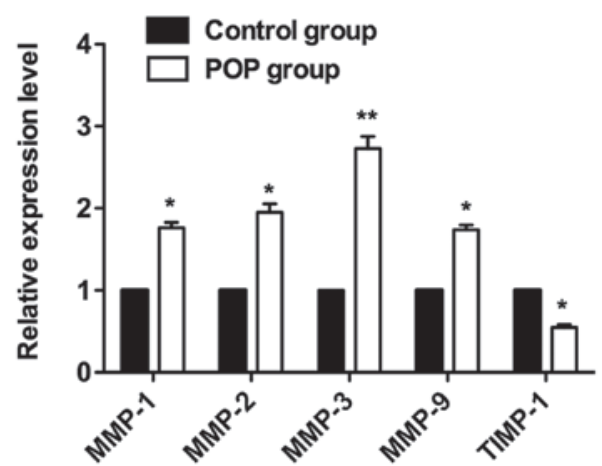

Figure 2. Polymerase chain reaction analysis of MMP and TIMP-1 expression levels in anterior vaginal wall tissues from POP patients and control group patients. Independent triplicate assays were performed for each RNA sample and data are shown as expression level fold-changes in the POP group relative to those in the control group, which were set as $1 .{ }^{*} \mathrm{P}<0.05,{ }^{* *} \mathrm{P}<0.005$ as compared with the control group. MMP, matrix metalloproteinase; POP, pelvic organ prolapse; TIMP-1, tissue inhibitor of metalloproteinase 1.

group, 12 of 72 patients had entered menopause and in the control group, 11 of 72 patients were menopausal. No statistical differences were identified in age, numbers of deliveries or menopausal status between the two groups $(\mathrm{P}>0.05$, Table II).

Expression levels of MMPs and TIMP-1 proteins evaluated by immunohistochemistry. Fig. 1 shows the intensity and the extent of MMP and TIMP-1 expression in the vaginal tissues from POP and control patients. MMPs and TIMPs exhibited cytoplasmic immunoreactivity in all tissue samples, but the staining intensities of the MMPs (MMP-1, 2, 3 and 9) in the POP group were significantly higher than those in the control group $(\mathrm{P}<0.05)$. By contrast, the staining intensity of TIMP-1 in the POP group was significantly lower than that in the control group $(\mathrm{P}<0.05)$.

Expression levels of MMP and TIMP-1 mRNA analyzed by $q P C R$. To validate the immunohistochemistry results, a qPCR assay was performed to analyze mRNA expression levels in the tissue samples. MMP and TIMP mRNA expression was 
Table III. Linear correlational analysis among gene expression levels.

\begin{tabular}{lcccc}
\hline Gene & MMP-2 (P-value/r value) & MMP-3 (P-value/r value) & MMP-9 (P-value/r value) & TIMP-1 (P-value/r value) \\
\hline MMP-2 & 1.000 & $0.006 / 0.865$ & $0.043 / 0.722$ & $0.065 /-0.678$ \\
MMP-3 & $0.006 / 0.865$ & 1.000 & $0.001 / 0.934$ & $0.000 /-0.944$ \\
MMP-9 & $0.043 / 0.722$ & $0.001 / 0.934$ & 1.000 & $0.003 /-0.891$ \\
TIMP-1 & $0.065 /-0.678$ & $0.000 /-0.944$ & $0.003 /-0.891$ & 1.000 \\
\hline
\end{tabular}

MMP, matrix metalloproteinase; TIMP, tissue inhibitor of metalloproteinase.

Table IV. Linear regression analysis among gene expression levels.

\begin{tabular}{lcccc}
\hline MMP-1 & R Square & $95 \%$ confidence interval & Intercept a & Regression coefficient b \\
\hline MMP-2 & 0.502 & $(-36.282,4.051)$ & -16.115 & 1.468 \\
MMP-3 & 0.703 & $(-67.843,-3.047)$ & -35.445 & 3.613 \\
MMP-9 & 0.504 & $(-20.720,8.820)$ & -5.950 & 1.219 \\
TIMP-1 & 0.614 & $(11.627,75.209)$ & 43.418 & -2.907 \\
\hline
\end{tabular}

MMP, matrix metalloproteinase; TIMP, tissue inhibitor of metalloproteinase.

detected in all vaginal tissue samples, although the expression levels varied among cases. The relative expression levels of MMP-1, -2, -3 and -9 mRNA in the vaginal tissues of the POP group were significantly higher than those of the control group (Fig. 2, $\mathrm{P}<0.05$ and $\mathrm{P}<0.005$ ). By contrast, the relative expression levels of TIPM-1 mRNA in the POP group were significantly lower than those in the control group $(\mathrm{P}<0.05)$.

Correlations among gene expression levels. The results that followed the normal distribution were then investigated by linear correlational analysis. As shown in Table III, significant positive correlations were identified among the expression levels of MMP-2, -3 and-9. The r correlation coefficients were: 0.865 between MMP-2 and MMP-3, 0.722 between MMP-2 and MMP-9, and 0.934 between MMP-3 and MMP-9. Conversely, significant negative correlations were detected between the expression levels of TIMP-1 and those of MMP-3 and MMP-9 ( $\mathrm{r}=-0.944$ and -0.891 , respectively). However, no significant correlation was identified between MMP-2 and TIMP-1 expression levels $(\mathrm{P}>0.05)$. The results that did not follow the normal distribution were investigated by linear regression analysis. As shown in Table IV, positive linear regression correlation was detected between the expression levels of MMP-1 and those of other MMPs $(\mathrm{P}<0.05)$, and negative linear regression correlation between the expression levels of MMP-1 and those of TIMP-1 $(\mathrm{P}<0.05)$.

\section{Discussion}

POP is a common disorder that affects the health and the life quality of elderly female patients. The understanding of pelvic floor disorders has advanced over the last few years. The etiology of POP is likely to be multifactorial; however, the biochemical features of the pelvic floor and the possible connective tissue alterations in patients with POP are not fully understood.

Collagen fiber is the main constituent of the pelvic floor fascia and ligaments. Collagen is also the main organizational structural component connecting the pelvic organs (6). A number of studies have reported that collagen is involved in the pathogenesis of POP, and these studies have mainly focused on the collagen content, morphology and metabolism $(7,8)$. The collagen content was considered to be reduced in the pelvic floor connective tissue of POP patients, with a reduction in the number of collagen fibers leading to the relaxation of the ligaments, fascia and other supportive structures, eventually resulting in the occurrence of POP. Further studies revealed that the collagen content in POP patients was significantly reduced, while the procollagen gene expression levels were not affected (15). The reduction in the collagen content in the pelvic floor tissue is thus considered to be due to increases in collagenase degradation. Changes in the ultrastructure and biochemical properties of collagen fibers have been demonstrated to be involved in the occurrence of POP (16).

MMPs are important enzymes that degrade collagen in the ECM. TIMPs inhibit the production and activation of MMPs, and thus inhibit the degradation of collagen. The imbalance between MMPs and TIMPs in ECM is closely associated with collagen metabolism (17). MMPs and TIMPs work together to increase the degradation of collagen in the pelvic floor connective tissue, leading to a reduction in collagen levels and the relaxation of the pelvic floor, eventually resulting in the occurrence of POP. Interstitial collagenase (MMP-1), gelatinase (MMP-2, MMP-9) and stromelysin (MMP-3) in pelvic tissue have also been found to be associated with the occurrence of POP. The expression levels of MMPs were observed to be 
increased, but the expression levels of TIMPs were demonstrated to be reduced in prolapse patients (18). Wu et al (19) found that MMP-9 expression levels in patients with POP were significantly higher than those in a control group, indicating that MMP-9 overexpression may be involved in the occurrence of POP. Strinic et al (20) have shown that the expression levels of MMP-1 and MMP-2 in POP patients were also significantly higher that those in a control group. Skorupski et al (21) reported that MMP-1 and MMP-3 expression levels in POP patients were markedly higher than those in healthy controls, suggesting that these levels were correlated with the incidence of POP. However, Gabriel et al (12) compared the expression levels of MMP-1 and MMP-2 in female patients with and without POP by immunohistochemistry and found that increased MMP-2 expression levels in the uterosacral ligament were associated with POP, while no significant difference in MMP-1 expression levels was identified between females with POP and healthy controls. As collagen regulatory factors, MMPs have attracted extensive attention. However, these previous studies observed somewhat conflicting results with regard to the expression levels of MMPs in POP, and accordingly, the understanding of the underlying mechanism of POP occurrence and development is limited.

In analogy to previous studies, in the present study, the differential expression of MMPs and TIMP-1 protein and mRNA in the anterior vaginal wall tissues from POP patients and control subjects was detected using immunohistochemistry and qPCR. The expression levels of MMP-1, -2, -3 and -9 mRNA and protein in the POP group were found to be markedly higher than those in the control group. By contrast, the expression levels of TIMP-1 mRNA and protein in the POP group were significantly lower than those in the control group. Correlational analysis revealed a positive correlation among MMP-2, -3 and -9 expression levels. TIMP-1 expression levels were negatively correlated with those of MMP-3 and -9 (Table III). Conversely, MMP-1 expression levels exhibited a positive correlation with those of the other MMPs (MMP-2, -3 and -9), but a negative correlation with TIMP-1 expression levels (Table IV). These findings demonstrated that the increased expression levels of MMPs and the reduced expression levels of TIMPs were directly associated with the presence of uterine prolapse, indicating that the differential expression of MMPs and TIMPs is associated with the occurrence and development of POP. Increased MMP expression levels and/or reduced TIMP-1 expression levels may result in a reduction in the collagen content in the vaginal wall connective tissue, resulting in damage to the pelvic floor structure, subsequently losing integrity and stability (22).

In conclusion, the expression levels of MMP-1, -2, -3 and -9 were demonstrated to be markedly increased, while TIMP-1 expression levels were decreased in the vaginal wall tissues from a POP group as compared with those from a control group. These findings suggested that the differential expression of MMPs and TIMPs was involved in the onset and development of POP. An effective way to treat POP may therefore be through the inhibition of MMP expression and increasing TIMP expression levels, which may inhibit the degradation of collagen and increase the local collagen content, thus enhancing tissue elasticity.

\section{Acknowledgements}

This study was supported by the National Natural Science Foundation of China (grant no. 81102713), the Key Program of Health Committee of Fujian Province of China (grant no. wzzz0911) and the Chen Keji Development Fund (grant no. CKJ2010006).

\section{References}

1. Weber AM and Richter HE: Pelvic organ prolapse. Obstet Gynecol 106: 615-634, 2005.

2. Goh JT: Biomechanical and biochemical assessments for pelvic organ prolapse. Curr Opin Obstet Gynecol 15: 391-394, 2003.

3. Suzme R, Yalcin O, Gurdol F, Gungor F and Bilir A: Connective tissue alterations in women with pelvic organ prolapse and urinary incontinence. Acta Obstet Gynecol Scand 86: 882-888, 2007.

4. Alperin M and Moalli PA: Remodeling of vaginal connective tissue in patients with prolapse. Curr Opin Obstet Gynecol 18: 544-550, 2006.

5. Gelse K, Pöschl E and Aigner T: Collagens - structure, function, and biosynthesis. Adv Drug Deliv Rev 55: 1531-1546, 2003.

6. Ewies AA, Al-Azzawi F and Thompson J: Changes in extracellular matrix proteins in the cardinal ligaments of post-menopausal women with or without prolapse: a computerized immunohistomorphometric analysis. Hum Reprod 18: 2189-2195, 2003.

7. Kerkhof MH, Hendriks L and Brölmann HA: Changes in connective tissue in patients with pelvic organ prolapse - a review of the current literature. Int Urogynecol J Pelvic Floor Dysfunct 20: 461-474, 2009.

8. Campeau L, Gorbachinsky I, Badlani GH and Andersson KE: Pelvic floor disorders: linking genetic risk factors to biochemical changes. BJU Int 108: 1240-1247, 2011.

9. Carmeliet P, Moons L, Lijnen R, et al: Urokinase-generated plasmin activates matrix metalloproteinases during aneurysm formation. Nat Genet 17: 439-444, 1997.

10. Amălinei C, Căruntu ID, Giuşcă SE and Bălan RA: Matrix metalloproteinases involvement in pathologic conditions. Rom J Morphol Embryol 51: 215-228, 2010.

11. Bourboulia D and Stetler-Stevenson WG: Matrix metalloproteinases (MMPs) and tissue inhibitors of metalloproteinases (TIMPs): Positive and negative regulators in tumor cell adhesion. Semin Cancer Biol 20: 161-168, 2010.

12. Gabriel B, Watermann D, Hancke K, et al: Increased expression of matrix metalloproteinase 2 in uterosacral ligaments is associated with pelvic organ prolapse. Int Urogynecol J Pelvic Floor Dysfunct 17: 478-482, 2006.

13. Jackson S, James M and Abrams P: The effect of oestradiol on vaginal collagen metabolism in postmenopausal women with genuine stress incontinence. BJOG 109: 339-344, 2002.

14. Phillips CH, Anthony F, Benyon C and Monga AK: Collagen metabolism in the uterosacral ligaments and vaginal skin of women with uterine prolapse. BJOG 113: 39-46, 2006.

15. Lammers K, Lince SL, Spath MA, et al: Pelvic organ prolapse and collagen-associated disorders. Int Urogynecol J 23: 313-319, 2012.

16. Johanes I, Mihelc E, Sivasankar M and Ivanisevic A: Morphological properties of collagen fibers in porcine lamina propria. J Voice 25: 254-257, 2011.

17. Gueders MM, Foidart JM, Noel A and Cataldo DD: Matrix metalloproteinases (MMPs) and tissue inhibitors of MMPs in the respiratory tract: potential implications in asthma and other lung diseases. Eur J Pharmacol 533: 133-144, 2006.

18. Chen BH, Wen Y, Li H and Polan ML: Collagen metabolism and turnover in women with stress urinary incontinence and pelvic prolapse. Int Urogynecol J Pelvic Floor Dysfunct 13: 80-87, 2002.

19. Wu JM, Visco AG, Grass EA, et al: Matrix metalloproteinase-9 genetic polymorphisms and the risk for advanced pelvic organ prolapse. Obstet Gynecol 120: 587-593, 2012.

20. Strinic T, Vulic M, Tomic S, et al: Matrix metalloproteinases-1, -2 expression in uterosacral ligaments from women with pelvic organ prolapse. Maturitas 64: 132-135, 2009.

21. Skorupski P, Jankiewicz K, Miotła P, et al: The polymorphisms of the MMP-1 and the MMP-3 genes and the risk of pelvic organ prolapse. Int Urogynecol J 24: 1033-1038, 2013.

22. Moore CS and Crocker SJ: An alternate perspective on the roles of TIMPs and MMPs in pathology. Am J Pathol 180: 12-16, 2012. 